\title{
Influência de elementos-traço na densidade mineral óssea de mulheres idosas
}

\author{
Trace elements influence on bone mineral density in elderly women \\ L. Lima, A.C. Furtado, R.M. Lima, V.M. Reis, R.M. Fonseca, R.J. Oliveira
}

ARTIGO ORIGINAL | ORIGINAL ARTICLE

\begin{abstract}
O objetivo do estudo foi comparar os níveis de elementos-traço (ET) de acordo com a densidade mineral óssea (DMO) em idosas. Participaram do estudo 27 idosas (65.70 \pm 3.96 anos). A amostra foi dividida em dois grupos homogêneos de acordo com a DMO, sendo o grupo 1 aquelas com maior DMO e no grupo 2 as com os menores valores. A DMO foi mensuradas utilizando o DEXA e os ET foram avaliados a partir de amostras de cabelo. No grupo 1 encontrou-se maiores teores de $\mathrm{Ca}, \mathrm{K}, \mathrm{Na}, \mathrm{Mo}, \mathrm{B}, \mathrm{Cu}$ e $\mathrm{Mg}$. Enquanto que no grupo 2 observou-se maiores concentrações de $\mathrm{Se}$ e o $\mathrm{Pb}$. Os resultados sugerem que o desequilíbrio na homeostase dos ET pode ser fator de risco para a redução da DMO e que as altas concentrações de $\mathrm{Pb}$ e Se no cabelo podem ser indicativos de redução da massa óssea.

Palavras-chave: envelhecimento, cabelo, estanho, chumbo
\end{abstract}

ABSTRACT

To compare trace elements (TE) levels according to bone mineral density (BMD) in elderly women. 27 elderly women $(65.7 \pm 3.96$ years $)$ were evaluated. They were classified in two groups: high BMD (group 1) and low BMD (group 2). BMD was measured in DXA and TE were evaluated from hair sample. Higher levels of $\mathrm{Ca}, \mathrm{K}, \mathrm{Na}, \mathrm{Mo}, \mathrm{B}, \mathrm{Cu}$ e $\mathrm{Mg}$ were found in group 1 (high $\mathrm{BMD}$ ) while Se and $\mathrm{Pb}$ were higher concentrated in group 2 (low $\mathrm{BMD}$ ). The results suggest that the imbalance in the homeostasis of ET may be a risk factor for reduced BMD and higher Pb and Se concentrations can mark bone mass loss.

Keywords: aging, hair, tin, lead

Submetido: 20.03.2012 | Aceite: 29.06.2013

Luciana Lima, Adriana C. Furtado. Universidade Católica de Brasília, Brasil.

Ricardo Moreno Lima, Romulo Maia Carlos Fonseca, Ricardo Jacó de Oliveira. Faculdade de Educação Fisica - Universidade de Brasilia, Brasil.

Victor Machado Reis. Universidade de Trás-os-Montes e Alto Douro, Portugal.

Endereço para correspondência: Ricardo Jacó de Oliveira, Universidade de Brasília, Campus Universitário Darcy Ribeiro, Faculdade de Educação Física, Asa Norte, Brasília, DF, CEP: 70910-970, Brasil.

E-mail: rjaco@unb.br 
O envelhecimento acarreta alterações na composição corporal, notada pelo declínio de massa muscular (sarcopenia) e aumento da gordura corporal. No entanto, a massa livre de gordura produz efeito positivo para o estímulo ósseo principalmente pela força de sustentação do corpo, evitando quedas no idoso (de Rekeneire et al., 2003). Conforme Kamel et al. (2002) a sarcopenia está associada à redução dos níveis de força muscular, decréscimo da área transversa, infiltração de tecido gorduroso e conectivo no músculo, além disso, ela também tem sido associada ao aumento do número de quedas, declínio da capacidade funcional e osteoporose (Kenney \& Buskirk, 1995).

Essas alterações relacionadas à composição corporal e funções orgânicas afetam a utilização e absorção de minerais (Mertz, 1996), também conhecidos como elementos-traço (Prentice, 2004). Ademais distúrbios nutricionais, em particular a deficiência de elementos-traço, estão associados ao envelhecimento (Gur et al., 2002; Okano, 1996). Uma doença comumente associada ao envelhecimento é a osteoporose, distúrbio osteometabólico caracterizado pela diminuição da densidade mineral óssea (DMO) levando a um aumento da fragilidade esquelética e do risco de fraturas, Como o tecido ósseo é constituído por matrizes orgânicas e inorgânicas, sendo formado principalmente por minerais, entende-se que alterações na absorção e utilização dos elementos-traço podem alterar o desenvolvimento e manutenção dessa matriz orgânica (Gur et al., 2002).

Nesse sentido, alguns autores (Berglund, Akesson, Bjellerup, \& Vahter, 2000; Brzoska, Moniuszko-Jakoniuk, Jurczuk, Galazyn-Sidorczuk, \& Rogalska, 2001; Jarup, Alfven, Persson, Toss, \& Elinder, 1998) sugerem que o cádmio, zinco, fosfato, chumbo, além de alguns outros elementos, à medida que se associam ao sangue, rins, túbulo gastrintestinal, por exemplo, alteram as concentrações de estruturas importantes para a homeostase da densidade mineral óssea. Além disso, os efeitos cumulativos de elementos-traço tóxicos, associados à restrição de elementos-traço essenciais acarretam prejuízo na captação mineral para o turnover ósseo.

Portanto, há a necessidade de se identificar o comportamento dos determinantes fisiológicos e ambientais que influenciam no risco de doenças relacionadas ao metabolismo ósseo. Contudo, a avaliação dos níveis dos elementos-traço pode possibilitar o diagnóstico precoce de algumas doenças e assim, medidas preventivas poderão ser tomadas a fim de evitar a osteoporose e, conseqüentemente, reduzir o risco de quedas e fraturas (Harris, 1993). Para tentar elucidar a questão, o presente estudo teve o propósito de comparar os níveis de elementos-traço (ET) de acordo com a densidade mineral óssea (DMO) em idosas.

\section{MÉTODO}

O presente estudo é caracterizado como uma pesquisa descritiva do tipo transversal. $\mathrm{O}$ estudo foi aprovado pelo Comitê de Ética em Pesquisa da Universidade Católica de Brasília (CEP/UCB n ${ }^{\circ}$ 077/2004) e a coleta foi iniciada logo após as voluntárias terem assinado o termo de consentimento livre e esclarecido.

\section{Amostra}

A amostra foi constituída por 27 voluntárias (idade: $65.7 \pm 3.96$ anos; massa corporal: $65.2 \pm 13.7 \mathrm{Kg}$; estatura: $152.3 \pm 6.5$ ) fisicamente ativas, residentes das cidades satélites do Distrito Federal, inscritas no "Projeto Geração de Ouro" da UCB, esse projeto visa integrar o idoso em atividades esportivas, recreativas e educacionais. Como critério de seleção, as voluntárias não poderiam ser portadores de câncer ou ter diabetes tipo I, doença de Alzheimer, quadro de desnutrição, prótese metálica, contato direto com produtos químicos (defensivos agrícolas) ou serem fumantes, fazerem ingestão frequente de álcool, terapia de reposição hormonal ou tintura recente no cabelo. A amostra foi dividida em dois grupos 
conforme os valores de DMO, sendo o grupo 1 aquelas classificadas com maior DMO e no grupo 2 as com os menores valores.

\section{Procedimentos}

Densitometria óssea: para determinação da DMO foi utilizado o aparelho de absorptiometria de raios X de dupla energia - DEXA Lunar ${ }^{\circledR}$, modelo DPX-IQ. Os pontos escolhidos para as medidas da DMO foram as vértebras L2-L4, colo do fêmur, trocânter e área de Wards.

Coleta das amostras de cabelo: as amostras de cabelo foram coletadas da região occipital entre 1 a $10 \mathrm{~mm}$ de distância do scalpo (couro cabeludo). Esta região é padronizada por ser menos susceptível à contaminação externa. Foram coletados 2 a 3 g de cabelo de cada voluntária (Pozebon, Dressler, \& Curtius, 1999 1999).

Lavagem e digestão: as amostras foram processadas de acordo com o método de lavagem desenvolvido pela International Atomic Energy Agency (IAEA). O cabelo foi lavado para a remoção de partículas de poeira, suor, gordura (Pozebon et al., 1999). O procedimento consistiu em três lavagens intercalando água ultra pura e acetona, com secagem em sistema de aquecimento brando a $70^{\circ} \mathrm{C}$ sob vácuo, utilizando liofilizador até massa constante, e armazenada em sacos plásticos. Antes da digestão, as amostras foram pesadas para a obtenção ou aproximação da massa a $0.3 \mathrm{~g}$. Em seguida, foram digeridas, utilizando $5.0 \mathrm{~mL}$ de ácido nítrico $\left(\mathrm{HNO}_{3}\right)$ ultra puro e $2.5 \mathrm{~mL}$ de peróxido de hidrogênio $\left(\mathrm{H}_{2} \mathrm{O}_{2}\right)$ com sistema de digestão por microondas para a abertura das amostras. O sistema de digestão consistiu em 30 ciclos com intervalos de 15 segundos em ácido nítrico $\left(\mathrm{HNO}_{3}\right)$ ultra puro e 25 ciclos com intervalos de 15 segundos em peróxido de hidrogênio $\left(\mathrm{H}_{2} \mathrm{O}_{2}\right)$, todos com irradiação a $125 \mathrm{~W}$. Posteriormente, o volume do digerido foi aumentado a $25 \mathrm{~mL}$ com água ultra pura (Dombovári \& Papp, 1998; Pozebon et al., 1999).

Análise das amostras: as concentrações de 28 elementos-traço - Prata (Ag), Alumínio (Al),
Boro (B), Bário (Ba), Berílio (Be), Cálcio (Ca), Cádmio $(\mathrm{Cd})$, Cromo $(\mathrm{Cr})$, Cobre $(\mathrm{Cu})$, Ferro $(\mathrm{Fe})$, Mercúrio $(\mathrm{Hg})$, Potássio (K), Lítio (Li), Magnésio $(\mathrm{Mg})$, Manganês ( $\mathrm{Mn})$, Molibdênio (Mo), Sódio (Na), Níquel (Ni), Chumbo $(\mathrm{Pb})$, Antimônio (Sb), Escândio (Sc), Selênio (Se), Estanho (Sn), Estrôncio (Sr), Titâneo (Ti), Ítrio $(\mathrm{Y})$, Zinco $(\mathrm{Zn})$, zirgônio $(\mathrm{Zr})$ - foram detectadas com a análise multi-elementar das amostras, realizada pela espectroscopia de emissão atômica por plasma de argônio induzido (ICPOES) e espectrometria de massa atômica (ICPMS, aparelho seqüencial ICP-AES ${ }^{\circledR}$ e SPECTR $^{\circledR}$ AA 220 FS). O processo analítico foi controlado pelo uso de uma matriz padrão.

\section{Análise Estatística}

A caracterização das amostras foram realizadas por médias e desvios-padrão. Os padrões de distribuição normal das variáveis DMO foram identificados pela prova de Kolmogorov-Smirnov. As inter-relações entre os sítios ósseos foram determinadas pelo coeficiente de correlação de Pearson. Uma análise por classificação hierárquica foi utilizada para separar a amostra em dois grupos homogêneos, com base na DMO dos sítios (L2-L4, colo do fêmur e trocânter) e área (triângulo de Wards). As amostras semelhantes foram agrupadas entre si. Para identificação das diferenças entre as médias das variáveis de DMO e da composição química dos ET, foi utilizada a análise de variância (ANOVA). O nível de significância foi definido como $p \leq .01$. Todas as análises estatísticas foram realizadas usando o programa SPSS 10.0.

\section{RESULTADOS}

A prova de Kolmogorov-Smirnov para a distribuição normal dos valores de DMO revelou que todas as distribuições dessa variável foram normais. Além disso, a correlação de Pearson demonstrou uma associação positiva entre todos os sítios ósseos analisados $(r \geq .76, p \leq .01)$, sendo que a correlação mais 
forte foi entre o colo do fêmur e triangulo de Wards $(r=.916 ; p=.01)$. A tabela 1 apresenta os resultados das concentrações dos ET obtidos pela análise com ICP-AES e ICP-MS em todas as amostras.

A partir da análise por classificação hierárquica, foi possível formar dois grupos de acordo com os valores da DMO, sendo que o grupo 1 apresentou valores médios de DMO significativamente maiores que o grupo 2 (tabela 2 ).

A tabela 3 apresenta a comparação entre os valores médios dos ET entre os dois grupos analisados. Somente os elementos Ag, B, Ca, $\mathrm{Cu}, \mathrm{K}, \mathrm{Mg}, \mathrm{Mo}, \mathrm{Na}, \mathrm{Pb}$ e Se apresentaram diferenças significativas entre os dois grupos, sendo que os elementos $\mathrm{Pb}$ e Se estavam em maior concentração no grupo 2 (com menor DMO).

\section{DISCUSSÃO}

Em se tratando da competição de elementos tóxicos e essenciais ou da quantidade desses afetando o osso, existe uma relação entre os

Tabela 1

Concentrações dos elementos-traço (ET) no cabelo de idosas. (média \pm desvio-padrão)

\begin{tabular}{cccccc}
\hline ET & \multicolumn{3}{c}{ ET } & ET & \\
\hline $\mathrm{Ag}$ & $0.24 \pm 0.29$ & $\mathrm{Hg}$ & $0.39 \pm 0.86$ & $\mathrm{Sc}$ & $0.671 \pm 2.534$ \\
$\mathrm{Al}$ & $15.53 \pm 40.45$ & $\mathrm{~K}$ & $43.80 \pm 69.13$ & $\mathrm{Se}$ & $6.290 \pm 6.780$ \\
$\mathrm{~B}$ & $1.00 \pm 3.22$ & $\mathrm{Li}$ & $13.27 \pm 23.49$ & $\mathrm{Te}$ & $0.50 \pm 1.001$ \\
$\mathrm{Ba}$ & $0.64 \pm 1.1$ & $\mathrm{Mg}$ & $26.362 \pm 44.116$ & $\mathrm{Sr}$ & $68.2 \pm 77.5$ \\
$\mathrm{Be}$ & $0.010 \pm 0.22$ & $\mathrm{Mn}$ & $4.86 \pm 5.157$ & $\mathrm{Ti}$ & $0.7 \pm 1.5$ \\
$\mathrm{Ca}$ & $760 \pm 731$ & $\mathrm{Mo}$ & $0.9 \pm 1.5$ & $\mathrm{Y}$ & $0.09 \pm 0.24$ \\
$\mathrm{Cd}$ & $0.156 \pm 0.215$ & $\mathrm{Na}$ & $683 \pm 670$ & $\mathrm{Zn}$ & $83.3 \pm 80.7$ \\
$\mathrm{Cr}$ & $4.604 \pm 5.935$ & $\mathrm{Ni}$ & $0.32 \pm 0.53$ & $\mathrm{Zr}$ & $5.7 \pm 7.61$ \\
$\mathrm{Cu}$ & $13.30 \pm 15.40$ & $\mathrm{~Pb}$ & $38.2 \pm 149.3$ & - & - \\
$\mathrm{Fe}$ & $10.40 \pm 17.91$ & $\mathrm{Sb}$ & $0.064 \pm 0.235$ & - & - \\
\hline
\end{tabular}

Nota: Os valores médios dos elementos foram determinados em $\mathrm{mg} / \mathrm{Kg}$. As, Co e V, não foram quantificados por essa metodologia.

Tabela 2

Densidade mineral óssea dos grupos formados após a classificação hierárquica (média \pm desvio-padrão)

\begin{tabular}{ccc}
\hline Variável & $\begin{array}{c}\text { Grupo } 1 \\
(n=15)\end{array}$ & $\begin{array}{c}\text { Grupo 2 } \\
(n=12)\end{array}$ \\
\hline Coluna Lombar $\left(\mathrm{g} / \mathrm{cm}^{2}\right)$ & $1.083 \pm 0.139$ & $0.808 \pm 0.103^{*}$ \\
Colo do fêmur $\left(\mathrm{g} / \mathrm{cm}^{2}\right)$ & $0.966 \pm 0.092$ & $0.674 \pm 0.071^{*}$ \\
Trocânter $\left(\mathrm{g} / \mathrm{cm}^{2}\right)$ & $0.849 \pm 0.095$ & $0.613 \pm 0.111^{*}$ \\
Area de Wards $\left(\mathrm{g} / \mathrm{cm}^{2}\right)$ & $0.790 \pm 0.108$ & $0.475 \pm 0.132^{*}$ \\
\hline
\end{tabular}

*Diferenças significativas entre os grupos $(p \leq .01)$.

Tabela 3

Comparação dos elementos-traço (ET) entre os grupos com maior DMO (grupo 1) e menor DMO (grupo 2)

\begin{tabular}{ccc}
\hline Elementos-traço* & $\begin{array}{c}\text { Grupo 1 } \\
(n=15)\end{array}$ & $\begin{array}{c}\text { Grupo 2 } \\
(n=12)\end{array}$ \\
\hline $\mathrm{Ag}$ & $0.04 \pm 0.04$ & $0.02 \pm 0.02$ \\
$\mathrm{~B}$ & $1.78 \pm 4.22$ & $0.40 \pm 0.92$ \\
$\mathrm{Ca}$ & $1012 \pm 874$ & $446 \pm 312$ \\
$\mathrm{Cu}$ & $16.27 \pm 17.62$ & $9.59 \pm 11.76$ \\
$\mathrm{~K}$ & $60.88 \pm 83.54$ & $22.43 \pm 38.94$ \\
$\mathrm{Mg}$ & $35.65 \pm 53.05$ & $14.76 \pm 27.47$ \\
$\mathrm{Mo}$ & $1.18 \pm 1.72$ & $0.52 \pm 0.94$ \\
$\mathrm{Na}$ & $823 \pm 74$ & $511 \pm 554$ \\
$\mathrm{~Pb}$ & $1.34 \pm 1.65$ & $84.06 \pm 220.27$ \\
$\mathrm{Se}$ & $4.98 \pm 5.78$ & $7.94 \pm 7.81$ \\
\hline
\end{tabular}

*Foram apresentados somente os ET que apresentaram diferenças significativas entre os grupos $(p \leq .01)$. 
ET e a característica do osso (cortical ou trabecular), o que independe do sítio analisado (Jurkiewicz, Wiechula, Nowak, \& Loska, 2005). Nesse estudo, uma correlação significante e positiva foi encontrada entre os sítios e a área analisados, demonstrando haver uma homogeneidade na interferência dos metais com a redução da massa óssea.

O cabelo funciona como um indicador biológico de envenenamento, deficiência de status nutricional e de algumas doenças crônicas como o câncer, doença de Parkinson e autismo, por ter afinidade com os elementos traço tóxicos (Batzevich, 1995; Morton, Carolan, \& Gardiner, 2002). No presente estudo, observou-se que as idosas com maior DMO (grupo 1) possuíam maiores concentrações dos minerais $\mathrm{Ag}, \mathrm{Ca}, \mathrm{K}$, $\mathrm{Na}, \mathrm{Mo}, \mathrm{B}, \mathrm{Cu}$ e $\mathrm{Mg}$ em relação ao outro grupo. $\mathrm{O} \mathrm{Ca}, \mathrm{Cu}$ e $\mathrm{Mg}$ são elementos que participam na formação óssea pela sua influência na remodelação óssea (Gur et al., 2002) e as maiores concentrações desses três ET encontradas no grupo com maior DMO reforça a importância desses elementos. Ademais, outros elementos como Ag, K, Na, Mo e B também estavam em maiores concentrações no grupo 1 , sugerindo que esses elementos também possam auxiliar o processo de formação da massa óssea.

Por outro lado, o $\mathrm{Pb}$ e o Se estavam em maiores concentrações no grupo com menor DMO (grupo 2). Doses sub-tóxicas de $\mathrm{Pb}$ podem modular a expressão e/ou atividade dos marcadores bioquímicos dos osteoblastos, o que torna $\mathrm{o} \mathrm{Pb}$ um bom parâmetro para identificar a mortalidade das células osteoblásticas (células de formação óssea) (Milgram, Carriere, Thiebault, Malaval, \& Gouget, 2008). Além de causar efeito nas células ósseas osteoblásticas, o $\mathrm{Pb}$ envolve-se com os condrócitos, inibindo o processo de formação endocondral, afetando o desenvolvimento esquelético (Gonzalez-Riola et al., 1997). Além disso, durante o envelhecimento, observa-se aumento da razão entre $\mathrm{Pb} /$ Ca na cabeça do fêmur, o que é corroborado com os resultados desse estudo, no qual foi observada uma relação de altas concentrações de $\mathrm{Pb}$ e baixa de $\mathrm{Ca}$ no grupo 2, demonstrando uma competição entre esses dois elementos e a presença de um efeito cumulativo do $\mathrm{Pb}$ no osso (Gerhardsson et al., 1993).

Já o Se, apesar de ser um elemento essencial, com função quelativa, torna-se tóxico em quantidades elevadas. Estudos em modelo animal (Turan, Balcik, \& Akkas, 1997; Turan, Bayari, Balcik, Severcan, \& Akkas, 2000) descreveram o efeito tanto da deficiência quanto do excesso de Se nas propriedades ósseas e demonstraram que, em ambos os casos, houve redução da resistência do osso nos grupos analisados. Essa redução da resistência deve-se à perda da rigidez e elasticidade estabelecida através da diminuição do conteúdo ósseo e da cristalinidade pela diminuição da razão Ca: $\mathrm{PO}_{4}$. Indiretamente, a presença de selênio em grandes quantidades pode causar o aumento do $\mathrm{Pb}$ e a redução de As, seu antagonista, gerando reação inversa à de proteção dos tecidos (Fulle et al., 2004) . Apesar de possuir um efeito protetor, no presente estudo foi demonstrado que o Se não evitou o aumento das concentrações de $\mathrm{Pb}$ no grupo de idosas com baixa DMO e seu excesso apesar de não ter causado intoxicação, contribuiu para a redução da massa óssea.

Portanto os resultados sugerem que tanto o excesso de $\mathrm{Pb}$ quanto de Se no cabelo possam ser indicadores de baixa DMO em mulheres idosas, porém há a necessidade de estudos experimentais para determinar o ponto de corte da concentração desses elementos e valores de osteopenia e osteoporose.

Pelo caráter transversal do estudo, os resultados aqui encontrados não explicam a relação causa-efeito e só poderão ser comprovados após a realização de estudos longitudinais, entretanto eles fornecem indícios sobre os efeitos dos elementos-traço na massa óssea de idosas e podem ser utilizados em próximos estudos. Em resumo, o presente estudo sugere que o desequilíbrio na homeostase dos ET pode ser fator de risco para a redução da DMO e que 
altas concentração dos elementos-traço $\mathrm{Pb}$ e Se no cabelo podem ser indicativos de redução da massa óssea. Sendo assim, a análise de cabelo apresenta-se com um método alternativo para identificar variações na DMO de idosos.

\section{CONCLUSÕES}

Tanto o excesso de $\mathrm{Pb}$ quanto o excesso de Se no cabelo parecem indicar baixa DMO em mulheres idosas. Há, no entanto, necessidade de estudos experimentais que determinem o ponto de corte da concentração desses elementos e os valores de osteopenia e osteoporose. O desequilíbrio na homeostase dos ET parece representar um fator de risco na redução da DMO e altas concentrações dos elementos-traço $\mathrm{Pb}$ e Se no cabelo podem ser indicativos de redução de massa óssea.

Agradecimentos:

Nada declarado.

Conflito de Interesses:

Nada declarado.

Financiamento:

Nada declarado.

\section{REFERÊNCIAS}

Batzevich, V. A. (1995). Hair trace element analysis in human ecology studies. The Science of the total environment, 164(2), 89-98.

Berglund, M., Akesson, A., Bjellerup, P., \& Vahter, M. (2000). Metal-bone interactions. Toxicology letters, 112-113, 219-225.

Brzoska, M. M., Moniuszko-Jakoniuk, J., Jurczuk, M., Galazyn-Sidorczuk, M., \& Rogalska, J. (2001). The effect of zinc supply on cadmium-induced changes in the tibia of rats. Food and chemical toxicology, 39(7), 729-737.

de Rekeneire, N., Visser, M., Peila, R., Nevitt, M. C., Cauley, J. A., Tylavsky, F. A., . . Harris, T. B. (2003). Is a fall just a fall: correlates of falling in healthy older persons. The Health, Aging and Body Composition Study. Journal of the American
Geriatrics Society, 51(6), 841-846.

Dombovári, J., \& Papp, L. (1998). Comparison of Sample Preparation Methods for Elemental Analysis of Human Hair. Microchemical Journal, 59(2), 187-193.

Fulle, S., Protasi, F., Di Tano, G., Pietrangelo, T., Beltramin, A., Boncompagni, S., . . . Fano, G. (2004). The contribution of reactive oxygen species to sarcopenia and muscle ageing. Experimental gerontology, 39(1), 17-24.

Gerhardsson, L., Attewell, R., Chettle, D. R., Englyst, V., Lundstrom, N. G., Nordberg, G. F., . . Todd, A. C. (1993). In vivo measurements of lead in bone in long-term exposed lead smelter workers. Archives of environmental health, 48(3), 147-156. doi: 10.1080/00039896.1993.9940813

Gonzalez-Riola, J., Hernandez, E. R., Escribano, A., Revilla, M., Ca, S., Villa, L. F., \& Rico, H. (1997). Effect of lead on bone and cartilage in sexually mature rats: a morphometric and histomorphometry study. Environmental research, 74(1), 91-93.

Gur, A., Colpan, L., Nas, K., Cevik, R., Sarac, J., Erdogan, F., \& Duz, M. Z. (2002). The role of trace minerals in the pathogenesis of postmenopausal osteoporosis and a new effect of calcitonin. Journal of bone and mineral metabolism, 20(1), 39-43. doi: 10.1007/s007740200005

Harris, S. T. (1993). Osteoporosis: pharmacologic treatment strategies. Advances in internal medicine, 38, 303-321.

Jarup, L., Alfven, T., Persson, B., Toss, G., \& Elinder, C. G. (1998). Cadmium may be a risk factor for osteoporosis. Occupational and environmental medicine, 55(7), 435-439.

Jurkiewicz, A., Wiechula, D., Nowak, R., \& Loska, K. (2005). Lead content in the femoral heads of inhabitants of Silesia. Journal of Trace Elements in Medicine \& Biology, 19(2-3), 165-170. doi: 10.1016/j.jtemb.2005.07.010

Kamel, H. K., Maas, D., \& Duthie, E. H., Jr. (2002). Role of hormones in the pathogenesis and management of sarcopenia. Drugs \& aging, 19(11), 865-877.

Kenney, W. L., \& Buskirk, E. R. (1995). Functional 
consequences of sarcopenia: effects on thermoregulation. The journals of gerontology. Series $A$, Biological sciences and medical sciences, 50, 78-85.

Mertz, W. (1996). Trace elements in the elderly. Nutrition, 12 (7-8), 549-550.

Milgram, S., Carriere, M., Thiebault, C., Malaval, L., \& Gouget, B. (2008). Cytotoxic and phenotypic effects of uranium and lead on osteoblastic cells are highly dependent on metal speciation. Toxicology, 250(1), 62-69. doi: 10.1016/j. tox.2008.06.003

Morton, J., Carolan, V., \& Gardiner, P. (2002). Removal of exogenously bound elements from human hair by various washing procedures and determination by inductively coupled plasma mass spectrometry. Analytica Chimica Acta, 455,(1), 23-34.

Okano, T. (1996). Effects of essential trace elements on bone turnover--in relation to the osteoporosis. Japanese journal of clinical medicine, 54(1), 148-154.
Pozebon, D., Dressler, V. L., \& Curtius, A. J. (1999). Análise de cabelo: uma revisão dos procedimentos para a determinação de elementos traço e aplicações. Química Nova, 22, 838-846.

Prentice, A. (2004). Diet, nutrition and the prevention of osteoporosis. Public health nutrition, 7(1A), 227-243.

Turan, B., Balcik, C., \& Akkas, N. (1997). Effect of dietary selenium and vitamin $\mathrm{E}$ on the biomechanical properties of rabbit bones. Clinical rheumatology, 16(5), 441-449.

Turan, B., Bayari, S., Balcik, C., Severcan, F., \& Akkas, N. (2000). A biomechanical and spectroscopic study of bone from rats with selenium deficiency and toxicity. Biometals, 13(2), 113-121.

\section{(cc) EY-NC} Todo o conteúdo da revista Motricidade está licenciado sob a Creative Commons, exceto quando especificado em contrário e nos conteúdos retirados de outras fontes bibliográficas. 